\section{VIDEOJUEGO PUEBLO PITANGA EN LA LUCHA CONTRA EL DENGUE EN COSTA RICA}

\section{VIDEOGAME "PITANGA TOWN" IN THE FIGHT AGAINST DENGUE IN COSTA RICA}

\author{
Antón Zamora ${ }^{1, a}$, Edén Galán-Rodas ${ }^{2, b}$, \\ Eduardo Ramírez ${ }^{3, c}$, Alfonso J. Rodríguez-Morales ${ }^{4, b}$, \\ Percy Mayta-Tristán ${ }^{5, \mathrm{~b}}$
}

Sr. Editor. Una de las prioridades en salud pública a nivel global es el control de las enfermedades transmitidas por vectores, como el dengue, la malaria, la enfermedad de Chagas, la leishmaniasis, la bartonelosis, entre otras. El dengue es una enfermedad viral transmitida por la picadura de las especies del mosquito Aedes, cuya incidencia global se ha incrementado rápidamente en las últimas décadas, estimándose que causa alrededor de 230 millones de infecciones cada año, de los cuales 25000 son mortales, localizándose principalmente en regiones tropicales y subtropicales ${ }^{(1)}$. La región de las Américas, durante el año 2013, tuvo el mayor reporte histórico de casos, con más de 2,3 millones de casos notificados por los países.

Al respecto, se están desarrollando diferentes iniciativas en el mundo que apuntan a mejorar en su diagnóstico, vigilancia epidemiológica, modelos de predicción y control, así como un mayor énfasis en la prevención y promoción de la salud (2,3); así, destacan experiencias que confirman la importancia del juego como elemento educativo y la utilidad de su aplicación práctica en la educación sanitaria (4). En este contexto, surge la iniciativa de promoción de la salud del Ministerio de Salud de Costa Rica, a través del videojuego "Pueblo Pitanga: enemigos silenciosos" inspirado en la dinámica de los videojuegos "The Secret of Monkey Island®" y "Paper Mario®", pero desarrollado con el objetivo de educar a la población joven sobre la higiene de una

\footnotetext{
Ministerio de Salud de Costa Rica. San José, Costa Rica.

Escuela de Medicina y Cirugía, Universidad Hispanoamericana. San José, Costa Rica.

3 Green Lava Studios. San José, Costa Rica.

4 Grupo de Investigación Salud Pública e Infección, Facultad de Ciencias de la Salud, Universidad Tecnológica de Pereira, Pereira. Risaralda, Colombia.

5 Escuela de Medicina, Universidad Peruana de Ciencias Aplicadas. Lima, Perú.

Informático médico; ${ }^{\mathrm{b}}$ médico salubrista; ${ }^{\mathrm{c}}$ ingeniero de sistemas Recibido: 23-03-15 Aprobado: 08-04-15
}

Citar como: Zamora A, Galán-Rodas E, Ramírez E, Rodríguez-Morales AJ, Mayta-Tristán P. Videojuego pueblo Pitanga en la lucha contra el dengue en Costa Rica [carta]. Rev Peru Med Exp Salud Publica. 2015;32(2):397-8. comunidad, y su relación con el control y la prevención de las enfermedades transmitidas por vectores, como el dengue ${ }^{(5)}$.

El videojuego se desarrolló desde finales de 2011 hasta abril de 2013. Durante este proceso se realizaron numerosas pruebas con jóvenes, conformados por 80 jugadores, divididos en grupos, con edades entre 9 y 16 años. A partir de los resultados obtenidos, se tomaron medidas para mejorar el nivel de entretenimiento del videojuego con el fin de garantizar su aceptación y usabilidad, mejorándolo constantemente en aspectos como el nivel de humor, la dinámica, el material educativo y la reusabilidad. Los resultados han sido muy positivos en cuanto al nivel de aprendizaje y diversión. En promedio, los jugadores pueden completar el videojuego en tres horas y media. El $100 \%$ de los jóvenes, evaluados posteriormente al uso del videojuego, reportaron una mejoría significativa en los cuestionarios de conocimiento realizados por nuestro equipo antes y después de jugar $(p<0,05)$; así también, encontraron el juego muy entretenido $y$, finalmente, expresaron que volverían a jugarlo. Además, pudimos observar que algunos de los elementos humorísticos podían mejorarse para alcanzar un mayor rango de edad y captar mejor la atención de los jugadores, lo cual nos permitió implementar estos cambios en la versión final.

Actualmente el videojuego se ha instalado en todas las escuelas y colegios de Costa Rica con un potencial acceso de 40000 estudiantes y está instalado en 144 centros informáticos comunales de acceso gratuito a la población; además, cuenta con 35000 descargas en Internet, y ha sido considerado por la Organización Panamericana de la Salud como una herramienta útil para educar y sensibilizar a la población joven sobre su papel en la prevención y control del dengue, esperando que su impacto se vea reflejado en un mayor control de la enfermedad y la disminución de su incidencia.

Finalmente, es importante destacar que el uso de las tecnologías de información y comunicación articuladas con una adecuada alfabetización en salud de la población, permiten desarrollar mayores potencialidades, considerando como una necesidad el empoderamiento de las personas respecto al autocuidado de su salud, adoptando conductas que promuevan estilos de vida saludables y una mejor calidad de vida.

Fuente de financiamiento: la Organización Panamericana de la Salud (OPS/OMS) financió el desarrollo del videojuego Pueblo Pitanga.

Conflictos de interés: los autores declaran no tener conflictos de interés con la publicación de este artículo. 


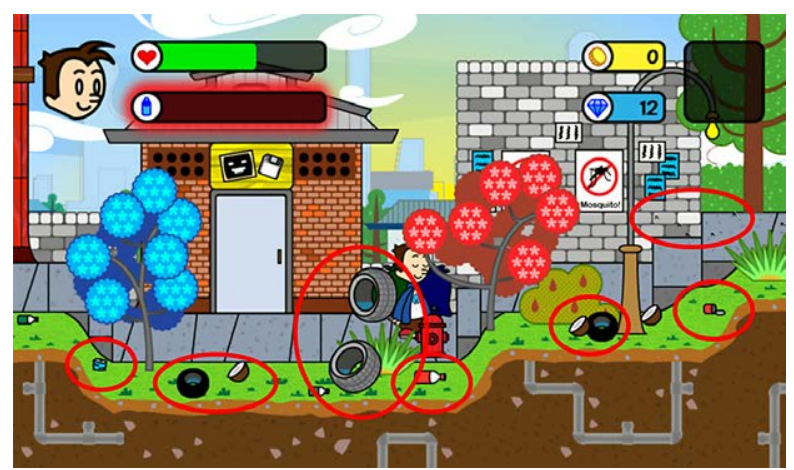

Figura 1. Imagen del juego donde se aprecian elementos ecoepidemiológicos relacionados con la transmisión del dengue (llantas o cauchos, botellas, latas y otros recipientes donde puede criarse el vector, así como los propios mosquitos) (link para el juego: http://www.pueblopitanga.com/).

Contribución de autoría: AZ, EGR y ER participaron en la concepción, diseño del estudio y recolección de los datos. Todos los autores participaron en el análisis e interpretación de los datos, en la redacción del borrador, en la revisión crítica del manuscrito y aprobaron la versión final a publicar.

\section{REFERENCIAS BIBLIOGRÁFICAS}

1. San Martin JL, Brathwaite O, Zambrano B, Solorzano JO, Bouckenooghe A, Dayan GH, et al. The epidemiology of dengue in the americas over the last three decades: a worrisome reality. Am J Trop Med Hyg. 2010 Jan;82(1):128-35. doi: 10.4269/ajtmh.2010.09-0346.

2. Oviedo E, Fernández A; Naciones Unidas. Salud electrónica en América Latina y el Caribe: avances y desafíos [Internet]. Santiago: CEPAL; 2010 [citado 15 de marzo de 2015]. Disponible en: http://repositorio.cepal.org/ handle/11362/32848

3. Wilder-Smith A, Renhorn KE, Tissera H, Abu Bakar S, Alphey L, Kittayapong P, et al. DengueTools: innovative tools and strategies for the surveillance and control of dengue. Glob Health Action. 2012;5. doi: 10.3402/gha.v5i0.17273.

4. Vivas E, Guevara de Sequeda M. Un juego como estrategia educativa para el control de Aedes aegypti en escolares venezolanos. Rev Panam Salud Pública. 2003 Dic;14(6):394-401.

5. Organización Panamericana de la Salud. ¡La prevención del Dengue es tarea de todos! - Pueblo Pitanga: Enemigos Silenciosos [Internet]. San José: OPS; 2013 [citado 15 de marzo de 2015]. Disponible en: http://www.paho.org/cor/ index.php?option $=$ com_content $\&$ view $=$ article $\&$ id $=173$ : ilaprevencion-del-dengue-es-tarea-de-todos-pueblo-pitangaenemigos-silenciosos\&catid=402: cor.03-epidemiologa,prevencin-y-control-de-da

Correspondencia: Edén Galán Rodas

Dirección: Av. 1 Calle 27. Apartado 11385. San José, Costa Rica.

Teléfono: +50683148834 .

Correo electrónico:edgarod6@gmail.com

\section{ASOCIACIÓN ENTRE NIVELES DE ALCOHOLEMIA PERMITIDOS Y MUERTES POR ACCIDENTES DE TRÁNSITO}

\section{ASSOCIATION BETWEEN PERMITTED LEVELS OF ALCOHOLEMIA AND TRAFFIC ACCIDENT DEATHS}

\author{
José Suclla-Velásquez ${ }^{1,2, a}$, Lutgarda Suclla-Velásquez ${ }^{1,2, b}$, \\ María Concha-Rondón ${ }^{1,2, b}$
}

Sr. Editor. Las lesiones por accidentes de tránsito son la octava causa de muerte en el mundo y primera causa de muerte en personas de 15 a 29 años. Por este motivo, la Asamblea General de las Naciones Unidas ha declarado como la Década de Acción por la Seguridad Vial al periodo 2011-2020 (1).

Se conocen cinco factores de riesgo importantes para accidentes de tránsito: velocidad, cascos, cinturones de seguridad, sistemas de retención infantil y alcohol al conducir, siendo este último uno de los más importantes. Ello ha ocasionado que la mayoría de países tengan niveles de alcoholemia máximos permitidos (1). No obstante, son pocos los estudios que evalúan la efectividad de esta medida. Por ello, realizamos un estudio ecológico para determinar cómo varía la tasa de muertes por accidentes de tránsito por cada 100000 habitantes de acuerdo al nivel de alcoholemia permitido.

La muestra fue de 105 países, incluyéndose a aquellos que figuran en el Reporte del Estado Global en Seguridad de Caminos 2013 de la Organización Mundial de la Salud (Global Status Report on Road Safety) y que tengan niveles de alcoholemia permitidos iguales para conductores noveles y profesionales. Se excluyeron países sin data disponible en dicho reporte.

Evaluamos el nivel de alcoholemia permitido (variable independiente) y la tasa de muertes por accidentes de tránsito por cada 100000 habitantes (variable dependiente), definiéndose muerte por accidente de tránsito a aquella ocurrida dentro de los 30 días posteriores al suceso.

\footnotetext{
1 Centro de Investigación y Estudios Médicos (CIEM). Arequipa, Perú.

2 Universidad Católica de Santa María. Arequipa, Perú.

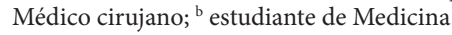

Recibido: 05-04-15 Aprobado: 22-04-15
}

Citar como: Suclla-Velásquez J, Suclla-Velásquez L, Concha-Rondón M. Asociación entre niveles de alcoholemia permitidos y muertes por accidentes de tránsito [carta]. Rev Peru Med Exp Salud Publica. 2015;32(2):398-9. 